\title{
Acidentes por escorpião em uma área do Nordeste de Amaralina, Salvador, Bahia, Brasil
}

\author{
Scorpion sting in an area of Nordeste de Amaralina, \\ Salvador, Bahia, Brazil. \\ Andréa Monteiro de Amorim ${ }^{1,3}$, Fernando Martins Carvalho ${ }^{2}$, \\ Rejâne Maria Lira-da-Silva ${ }^{3}$ e Tania Kobler Brazil ${ }^{3}$
}

\begin{abstract}
Resumo Um estudo epidemiológico mediu a prevalência de pessoas que referiram acidentes por escorpião em uma amostra populacional do Areal, bairro Nordeste de Amaralina, Salvador, Bahia. Examinou-se uma amostra aleatória sistemática de 1367 indivíduos, correspondendo a 44,4\% da população da área. Oitenta e dois indivíduos referiram haver sido picados por escorpião desde que residiam no Areal, resultando numa prevalência de $6 \%$ (IC 95\% 4,7 - 7,3). A prevalência de pessoas picadas por escorpião aumentou nos grupos com maior tempo de residência no domicílio e com maior idade atual. Chamou atenção que $92,7 \%$ dos acidentes aconteceram dentro do domicílio. A incidência estimada para o período mais recente (janeiro a julho de 2000) foi de 1,15 casos/1.000 habitantes por mês. Este coeficiente compara-se ao mais elevado já referido na literatura especializada para uma área epidêmica para acidentes escorpiônicos.
\end{abstract}

Palavras-chaves: Acidentes por escorpião. Prevalência. Incidência. Inquérito domiciliar.

Abstract An epidemiological study was undertaken to determine the prevalence of individuals who referred scorpion sting accidents in a population sample from Areal, a neighborhood northeast of Amaralina, Salvador City, State of Bahia, Brazil. A random, systematic sample of 1,367 individuals was taken, corresponding to $44.4 \%$ of the total population. Eighty-two residents referred scorpion sting since they were resident in Areal, giving a prevalence coefficient of $6 \%(95 \% \mathrm{Cl} 4.7-7.3)$. The prevalence of persons stung by scorpions increased according to greater time spent in the domicile and more advanced age. It was remarkable that $92.7 \%$ of the scorpions stings occurred within the home. The incidence coefficient estimated for the most recent period of time (January to July, 2000) was 1.15 cases/1,000 inhabitants per month, comparable to the highest ever reported for an epidemic area.

Key-words: Scorpion stings. Prevalence. Incidence. Survey.

O escorpionismo é reconhecido como um problema de Saúde Pública em vários países em desenvolvimento, como Marrocos ${ }^{2122}$, Tunísia ${ }^{13}$, México ${ }^{4}$ e Venezuela ${ }^{10}$, onde são importante causa de morbi-mortalidade. No Brasil, estima-se que ocorram cerca de 8.000 acidentes/ano, representando uma incidência anual aproximada de 3 casos $/ 100.000$ habitantes, estando $50 \%$ deles restritos aos Estados de Minas Gerais e São Paulo'. Recentemente, foi observado um aumento das notificações nos Estados da Bahia, Rio Grande do Norte, Alagoas e Ceará12.
A quase totalidade dos estudos sobre escorpionismo refere-se à descrição dos acidentes e à severidade dos casos ocorridos em populações hospitalares altamente selecionadas 24781317202428 . São escassos os estudos epidemiológicos, de base populacional definida, sobre o escorpionismo em áreas urbanas consideradas endêmicas para este agravo, e que também investiguem características dos agentes etiológicos responsáveis pelo acidente. Dentre os estudos com este escopo, destacamse os trabalhos de Spirandelli-Cruz et $\mathrm{al}^{26}$, desenvolvido

\footnotetext{
1. Programa Integrado de Saúde Ambiental e do Trabalhador do Instituto de Saúde Coletiva, Salvador, BA. 2. Departamento de Medicina Preventiva da Faculdade de Medicina da Universidade Federal da Bahia, Salvador, BA. 3. Núcleo de Ofiologia e Animais Peçonhentos do Departamento de Zoologia do Instituto de Biologia da Universidade Federal de Bahia, Salvador, BA.

Endereço para correspondência: Dra. Andréa Monteiro de Amorim. Av. Paulo VI s/nº, Ed. Uruguai/303, Pituba, 41810-000 Salvador, BA.

Tel: $71358-8032$.

e-mail:amoa@ufba.br

Recebido para publicação em 26/11/2001.
} 
em uma área endêmica do Estado de São Paulo e o de De Sousa et $\mathrm{al}^{8}$, realizado no Estado de Sucre, Venezuela.

Objetivou-se determinar a prevalência de pessoas picadas por escorpião em uma amostra populacional do Areal, bairro do Nordeste de Amaralina, Salvador, Bahia, e descrever a variação desta prevalência segundo algumas características sócio-demográficas dos moradores.

\section{MATERIAL E MÉTODOS}

Área de estudo. Esta pesquisa foi desenvolvida na área geográfica delimitada pelos Setores Censitários (SC) 64 e 68, pertencentes à Zona de Informação (ZI) 07, Distrito Sanitário Barra - Rio Vermelho, Subdistrito de Amaralina ${ }^{6}$. Estes Setores Censitários pertencem ao núcleo denominado Areal, no bairro do Nordeste de Amaralina, Salvador, Bahia.

População do estudo. Moradores da área de estudo, totalizando 3.080 pessoas, residentes em 806 domicílios $^{14}$. Desta população foi retirada uma amostra aleatória sistemática por blocos, onde a cada bloco de 10 casas, três foram sorteadas. Cerca de 10 domicílios sem respondentes devido a recusas, casa em reforma ou fechada foram imediatamente repostos por um novo sorteio.

Um estudo piloto foi conduzido na área, resultando numa prevalência de acidente escorpiônico de $P=7,5 \%$. Com base nesta estimativa, calculou-se a amostra seguindo as recomendações de Lwanga \& Lemeshow ${ }^{23}$ onde, para se obter uma precisão relativa de $25 \%$ e um $a=0,05$, seria necessário uma amostra de 758 indivíduos.

Fonte de dados. As informações obtidas nas entrevistas domiciliares, realizadas entre abril e julho de 2000, por uma equipe de sete entrevistadores previamente treinados, foram reunidas em fichas padronizadas. Foi realizado um estudo piloto para padronizar a forma de abordagem da população pelos entrevistadores. Os procedimentos utilizados para garantir a qualidade dos dados incluíram ainda a supervisão no decorrer das entrevistas e a revisão dos registros, antes das informações serem incluídas no banco de dados. A aplicação dos questionários foi realizada na área de estudo, onde os entrevistadores realizaram o inquérito nas residências selecionadas. As perguntas foram feitas a um dos membros da família, preferencialmente à dona da casa.

Prevalência de pessoas picadas por escorpião (acidente escorpiônico). O numerador foi considerado como o número de pessoas que referiram o acidente em algum tempo de suas vidas, enquanto residiam no Areal e o denominador foi o número de pessoas amostradas da população residente no Areal em abriljulho de 2000 (período de execução do trabalho de campo). A ocorrência do evento (acidente escorpiônico) foi referida para todo o período de tempo em que cada indivíduo entrevistado morou no domicílio atual.

Características da população. foram consideradas as variáveis: tempo de residência no domicílio onde houve a entrevista (domicílio atual) e grupos de idade atual, categorizada em anos; sexo; inserção atual no mercado de trabalho (assalariado=indivíduos que trabalhavam com carteira assinada, autônomo; do lar não remunerado; menor de 10 anos não estudante; estudante; desempregado/aposentado; autônomo e estudante; assalariado e estudante); grau de escolaridade atual (ensino fundamental $\mathrm{I}=$ educação infantil à $4^{\mathrm{a}}$ série do ensino básico, ensino fundamental $\|=5^{\text {a }}$ à $8^{\underline{a}}$ série do ensino básico, ensino médio $=1^{\circ}$ ao $3^{\circ}$ ano do ensino médio, ensino superior, nunca estudou, menores de 7 anos); tempo atual de permanência no domicílio (manhã, tarde, noite, dois turnos, três turnos, outra opção=trabalhadores de turno variável). Para cada uma dessas variáveis foram realizadas as medidas de ocorrência (freqüência simples e prevalência). O ano do acidente foi referido pelos indivíduos acidentados.

Processamento dos dados. As medidas descritivas básicas utilizadas para as análises foram: freqüência simples, média aritmética e mediana, desvio padrão, coeficientes de incidência e de prevalência e seus respectivos intervalos de $95 \%$ de confiança. Para as inferências estatísticas foram utilizados, quando indicados, testes de diferenças de médias (normal reduzida "Z" ou testes "t" para amostras independentes, testes bicaudais) e entre proporções (teste $X^{2}$ ). Os dados foram analisados com auxílio do programa SPSS® for Windows - Statistical Package for the Social Sciences) ${ }^{27}$.

\section{RESULTADOS}

Foram entrevistados 1367 indivíduos residentes em 306 domicílios situados na área do estudo, correspondendo a $44,4 \%$ do total da população residente e a $38 \%$ dos domicílios existentes nessa localidade. Oitenta e dois indivíduos referiram terem sido picados por escorpião desde que residiam no Areal, resultando no coeficiente de prevalência de $6 \%$ (IC 95\% 4,7-7,3). A precisão relativa deste coeficiente foi $21 \%$, recalculada com base no tamanho da amostra efetivamente examinada.

O tempo de residência no Areal variou de 70 anos (desde 1930) a menos de um ano (2000). Dos 82 acidentados, $76(92,7 \%)$ foram picados na residência atual, enquanto seis pessoas foram picadas no Areal, porém em outro ambiente (via pública, bar, escola, residência de outra pessoa ou na antiga casa). No total, foram referidos 87 acidentes escorpiônicos (eventos) domiciliares, sendo que 67 indivíduos foram picados uma única vez, sete foram acidentados duas vezes e apenas duas pessoas referiram ter sido acidentadas três vezes na sua residência.

A média do tempo de residência no domicílio onde aconteceu a entrevista (domicílio atual) foi maior 
( $p<0,0001$ ) entre os 80 acidentados do que entre os 1260 indivíduos que não sofreram acidente:21,3 $\pm 12,1$ anos e 12,8 $\pm 12,1$ anos, respectivamente. A prevalência da referência ao acidente escorpiônico aumentou progressivamente $(p<0,0001)$ de $0,8 \%$ para $33,3 \%$, segundo o aumento do tempo de residência no domicílio atual (0 a 69 anos) (Tabela 1).

A idade atual dos indivíduos entrevistados variou de 0 a 93 anos, tendo a maior parte da população entre 15 e $52(62,1 \%)$ anos. A média de idade foi maior $(P<0,0001)$ entre os 82 acidentados do que entre os

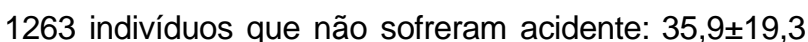
anos e 26,9 $\pm 20,0$ anos, respectivamente. Observou-se um aumento progressivo da prevalência da referência ao acidente escorpiônico $(p<0,0001)$ de $0,0 \%$ para $12,5 \%$ segundo o aumento da idade, classificados de acordo com os grupos apresentados na Tabela 2.

A prevalência de pessoas picadas por escorpião foi semelhante $(p>0,05)$ no sexo masculino 6,0\% $(38 / 631)$ e no feminino 5,2\% (38/736). Dos 76 acidentados, 32 (42\%) referiram que procuraram algum serviço de saúde. Destes 32 indivíduos, 21 (65,6\%) eram do sexo feminino.

\begin{tabular}{|c|c|c|c|}
\hline \multirow[t]{2}{*}{$\begin{array}{l}\text { Tempo de residência } \\
\text { no domicílio (anos)* }\end{array}$} & \multirow[t]{2}{*}{ Residentes $\mathrm{n}^{0}$} & \multicolumn{2}{|c|}{ Prevalência } \\
\hline & & $\mathrm{n}^{0}$ & $\%$ \\
\hline $0-1$ & 238 & 2 & 0,8 \\
\hline $2-6$ & 281 & 8 & 2,8 \\
\hline $7-14$ & 300 & 14 & 4,7 \\
\hline $15-24$ & 283 & 30 & 10,6 \\
\hline $25-52$ & 235 & 25 & 10,6 \\
\hline $53-69$ & 3 & 1 & 33,3 \\
\hline Total & 1.340 & 80 & 6,0 \\
\hline
\end{tabular}

\begin{tabular}{|c|c|c|c|}
\hline \multirow[t]{2}{*}{ Idade atual (anos)* } & \multirow{2}{*}{$\begin{array}{c}\text { Residentes } \\
(\mathrm{n} \div 2)\end{array}$} & \multicolumn{2}{|c|}{ Prevalência } \\
\hline & & $\mathrm{n}-0$ & $\%$ \\
\hline $0-1$ & 56 & 0 & 0,0 \\
\hline $2-6$ & 125 & 0 & 0,0 \\
\hline $7-14$ & 192 & 6 & 3,1 \\
\hline $15-24$ & 351 & 22 & 6,3 \\
\hline $25-52$ & 485 & 37 & 7,6 \\
\hline $53-93$ & 136 & 17 & 12,5 \\
\hline Total & 1.345 & 82 & 6,1 \\
\hline
\end{tabular}

Observou-se que $65,7 \%$ da população residente pertencia às categorias estudante, assalariado e desempregado/aposentado. A maior prevalência de acidentes escorpiônicos foi no grupo de assalariado+estudante $(12,5 \%)$, seguido pelos do lar não remunerados $(9,4 \%)$ e pelos desempregados/ aposentados (8,3\%).

Nas categorias Ensino Fundamental I e II encontrava-se $61,6 \%$ da população. No entanto, a maior prevalência dos acidentes escorpiônicos aconteceu no grupo pertencente à categoria de Ensino Médio (9,4\%), seguido pelos que nunca estudaram $(9,0 \%)$.

Notou-se que a maioria da população amostrada $(96,9 \%)$ permanecia em casa à noite, em dois turnos ou nos três turnos do dia. No entanto, a maior prevalência de acidentes ocorreu entre aqueles que permaneciam no domicílio apenas pela manhã (16,7\%).

Os 71 acidentes referidos aconteceram entre 1980 e 2000, com exceção dos anos 1981, 1983, 1985, 1987 a 1989 e 1991. A partir de 1992, houve casos de acidente em todos os anos, com maior freqüência em 1996 (14,1\%), 1999 (12,7\%), 1995 e 1997 (11,3\%, para cada). No período de janeiro a julho/2000, foi referida a freqüência mais elevada de acidentes escorpiônicos (15,5\%) (Tabela 3). Desta forma, uma estimativa de incidência para o período mais recente de janeiro a julho de 2000 , forneceria 0 coeficiente 8,05 casos/1000 habitantes em sete meses, ou seja, 1,15 casos/1000 habitantes por mês (IC 95\%: $0,58-1,71)$. 


\begin{tabular}{lrc}
\multicolumn{3}{c}{ Tabela 3 - Freqüencia absoluta e relativa (\%) de acidente } \\
escorpiônico domiciliar segundo o ano de ocorrência \\
(Areal, Nordeste de Amaralina, Salvador, Bahia, 2000). \\
\hline Ano* & \multicolumn{2}{c}{ Acidente escorpiônico } \\
\cline { 2 - 3 } & no & $\%$ \\
\hline 1980 & 1 & 1,4 \\
1982 & 2 & 2,8 \\
1984 & 1 & 1,4 \\
1986 & 1 & 1,4 \\
1990 & 4 & 5,6 \\
1992 & 6 & 8,5 \\
1993 & 2 & 2,8 \\
1994 & 3 & 4,2 \\
1995 & 8 & 11,3 \\
1996 & 10 & 14,1 \\
1997 & 8 & 11,3 \\
1998 & 5 & 7,0 \\
1999 & 9 & 12,7 \\
$2000^{* *}$ & 11 & 15,5 \\
\hline Total & 71 & 100,0 \\
\hline 5
\end{tabular}

* 5 casos sem informação para o ano do acidente.

** Período janeiro a julho de 2000.

\section{DISCUSSÃO}

Os acidentes por escorpião ocorrem muito freqüentemente na área estudada, considerando a magnitude dos coeficientes de prevalência ou de incidência estimados. A prevalência de pessoas picadas por escorpião encontrada no presente estudo (6 casos por 100 habitantes, IC95\% 4,7 - 7,3) é a maior já relatada, quando comparada a áreas consideradas hiperendêmicas para o escorpionismo. Franco \& Jaime ${ }^{11}$ descreveram uma incidência anual de 13,5/1000 habitantes no ano de 1955, na cidade de Durango (México) e De Sousa et $a^{8}$ referiram uma incidência de 68,3/10.000 na localidade de São Fernando (Venezuela) no triênio 1989-1991. No Brasil, Spirandeli-Cruz et al ${ }^{26}$ relataram uma incidência anual de 43 casos/100.000 habitantes, em 1991, para a microrregião administrativa do Vale do Paraíba (SP).

Na literatura consultada, a maior parte dos autores denominou de incidência a ocorrência dos acidentes escorpiônicos. Sem dúvida, acidentes são eventos agudos, de início claramente definido e que se encaixam perfeitamente sob a denominação de casos novos, que comporiam o numerador de uma medida de incidência. Entretanto, todos os estudos aqui citados, pecaram por não definir satisfatoriamente o denominador desta medida de incidência: - Qual seria a população exposta ao risco destes acidentes? Deste modo, no presente estudo optou-se por descrever o escorpionismo segundo a medida prevalência de pessoas picadas e não segundo a incidência, devido à escassez de informações sobre a alta mobilidade da população da área. Por estes motivos, fez-se uma estimativa menos enviesada para a incidência referente a um período mais curto e recente (janeiro-julho 2000): 1,15 casos/1.000 habitantes por mês. Este valor confirma o Areal como uma das localidades com incidência de acidente escorpiônico mais elevadas referidas na literatura.

$\mathrm{Na}$ amostra populacional estudada no Areal observou-se uma relativamente baixa recorrência, no mesmo indivíduo, de acidentes escorpiônicos (11,8\%). Este fato pode decorrer da alta mobilidade da população residente no bairro Nordeste de Amaralina. Para algumas localidades do México, foram relatadas altas reincidências de acidente escorpiônico, onde até $78 \%$ dos casos reportados no período de um ano foram picados mais de uma vez ${ }^{428}$.

A maior ocorrência de acidentes por escorpião no interior dos domicílios (92,3\%), encontrada neste estudo, é uma característica também referida por outros autores ${ }^{13} 25$. Justificativas para este fato foram dadas por diferentes autores que sugeriram uma relação entre a maior incidência destes acidentes em área domiciliar com o desemprego, que levaria os indivíduos a permanecer mais tempo em suas residências ${ }^{2}$, ou com o serviço doméstico, pois os escorpiões se adaptavam facilmente ao domicílio, onde aconteciam estes acidentes ${ }^{318}$. Nossos achados, porém, não podem confirmar estes dados pois a maior prevalência encontrada foi no grupo de pessoas que passavam a maior parte do tempo fora do ambiente doméstico (assalariado+estudante, 12,5\%). No entanto, agrupando os desempregados/aposentados e os do lar não remunerados encontrou-se uma prevalência de $17,7 \%$ de indivíduos acidentados, o que concordaria com os autores citados.

A prevalência de pessoas vítimas de acidente escorpiônico foi ligeiramente superior $(6 \%)$ para os homens que para as mulheres $(5,2 \%)$. Isto concorda com os dados descritivos das casuísticas de diversos 
autores para o Brasill 2171920 , México ${ }^{48}$ e Venezuela ${ }^{24}$. Franco \& Jaime ${ }^{11}$, no entanto, relataram uma maior proporção de mulheres acidentadas, justificando este dado à maior permanência das mulheres dentro de suas residências, estando mais expostas a este evento.

Embora a maior parte da literatura citada se referisse à maior ocorrência de acidentes entre os homens, estes dados poderiam estar relacionados à procura de atendimento médico. Entretanto, na amostra estudada no Areal, $65,6 \%$ dos acidentados que procuraram algum serviço de saúde eram do sexo feminino, apesar da ocorrência de acidentes no sexo masculino ter sido mais freqüente. Esta informação pode ser compreendida pelo fato de que tipicamente, as mulheres apresentam maior consumo de consultas médicas do que os homens ${ }^{515}$.

A coleta de dados desta pesquisa aconteceu entre abril e julho de 2000 e este foi o ano em que os entrevistados referiram o maior número de acidentes escorpiônicos (15,5\%). Isto pode estar relacionado ao viés de memória dos indivíduos acidentados, fato comum em estudos com características retrospectivas ${ }^{16}$. Através da coleta de dados secundários, realizado pela autora (AM Amorim: dados não publicados, 2001), no Centro de Informação Anti-Veneno da Bahia (CIAVE), observou-se que foram atendidos 123 casos de acidente escorpiônico com identificação do agente etiológico, para o período de 1982 a junho de 2000 , ocorridos no
Nordeste de Amaralina, sendo os anos de 1990 (9,8\%), 1994 (9,8\%), 1995 (15,4\%), 1996 (17,1\%) e 1997 $(12,2 \%)$ os que apresentaram maior freqüência de notificações. Pôde-se observar ainda que para os anos 1983 e 1986 não houve notificações de acidentes escorpiônicos provenientes do Nordeste de Amaralina no CIAVE; para 1985, 1988 e 1989 estes eventos não foram registrados neste Centro nem referidos pelos acidentados entrevistados neste trabalho. Vale ressaltar que nos anos de 1988 e 1989, o CIAVE esteve com atendimento restrito, devendo-se a isso a falta de notificações neste período. Os anos de 1985, 1986 e 1987 foram períodos atípicos, devido ao aumento das notificações pelas Diretorias Regionais de Saúde (DIRES) do Estado por causa da falta de soros antivenenos no país, o que forçou estas instituições a procurarem o CIAVE na busca de soluções para este problema, sendo portanto anos que apresentaram um quadro mais real do escorpionismo na nossa região ${ }^{17}$.

Concluiu-se que o instrumental metodológico da Epidemiologia pode contribuir substancialmente para um adequado dimensionamento da prevalência dos acidentes escorpiônicos numa dada população e que a incidência estimada de 1,15/1000 habitantes ao mês é a mais elevada já referida na literatura, caracterizando a área estudada como endêmica para acidentes por escorpião.

\section{REFERÊNCIAS BIBLIOGRÁFICAS}

1. Araújo FAA, Resende CC. Escorpionismo no Brasil - 1988/1989. Fundação Nacional de Saúde/Ministério da Saúde/Programa Nacional de Ofidismo. Relatório. 1990.

2. Biondi-de-Queiroz I. Escorpionismo no Estado da Bahia: estudo epidemiológico e clínico dos acidentes atendidos no Centro de Informação Anti-Veneno (CIAVE), no período de 1995-1997. Dissertação de Mestrado, Universidade Estadual de Feira de Santana, Feira de Santana, BA, 1999.

3. Bücherl W. Escorpionismo no Brasil. Memórias do Instituto Butantan 34: 9-24, 1969.

4. Calderón-Aranda ES, Dehesa-Dávila M, Chavez-Haro A, Possani LD. Scorpions stings and their treatment in Mexico. Envenomings and their treatments p. 311-320. Marcel Mérieux, 1996.

5. Carvalho FM, Silvany-Neto AM, Paim JS, Melo AMC, Azaro MGA. Morbidade referida e utilização de consulta médica em cinco populações do Estado da Bahia. Ciência e Cultura 40:853-858, 1988.

6. Companhia de Desenvolvimento da Região Metropolitana de Salvador/ CONDER. Mapa dos Setores Censitários 64 e 68, Zona de Informação 07, Subdistrito de Amaralina, Salvador, Bahia, 1992.

7. Dehesa-Dávila M. Epidemiological characteristics of scorpion sting in Léon, Guanajuato, México. Toxicon 27: 281-289, 1989.

8. De Sousa L, Bonoli S, Quiroga M, Parrila P. Scorpion sting epidemiology in Montes Municipality of State of Sucre, Venezuela: geographic distribution (1). Revista do Instituto de Medicina Tropical de São Paulo 38:147-152, 1996.

9. De Sousa L, Parrila P, Tillero L, Valdiviezo A, Jorquera A, Quiroga M. Scorpion poisoning in the Acosta and Caripe Counties of Monagas State, Venezuela. Part 1: Characterization of some epidemiological aspects. Cadernos de Saúde Pública, Rio de Janeiro 13: 45-51, 1997.

10. De Sousa L, Parrila-Alvarez P, Quiroga M. An epidemiological review of scorpion stings in Venezuela: the Northeastern Region. Journal of Venomous Animals and Toxins 6: 1-31, 2000.

11. Franco LV, Jaime ML. Consideraciones epidemiologicas sobre la picadura por alacran en la ciudad de Durango. Revista Invest. Salud Pública (México) Vol. XXVI. 1:7-21, 1996.

12. Fundação Nacional de Saúde. Manual de diagnóstico e tratamento de acidentes por animais peçonhentos. Ministério da Saúde. 1999.

13. Goyffon M, Vachon M, Broglio N. Epidemiological and clinical characteristics of the scorpion envenomation in Tunisia. Toxicon 20: 337-344, 1982.

14. Instituto Brasileiro de Geografia e Estatística/Bahia. Dados populacionais dos Setores Censitários 64 e 68, Zona de Informação 07, Subdistrito de Amaralina, Censo Demográfico Parcial/1996, Salvador, Bahia, 1996.

15. Kohn R, White KL. Health care: an international study. Oxford, Oxford University Press, 1976.

16. Kroeger A. Errores de respuestas y otros problemas de las encuestas de salud mediante entrevista en los países em desarollo. Boletin Oficina Sanitaria Panamericana 100: 253-282, 1986.

17. Lira-da-Silva RM, Amorim AM, Brazil TK. Envenenamento por Tityus stigmurus na Região Metropolitana de Salvador, Bahia, 
Brasil. Revista da Sociedade Brasileira de Medicina Tropical 33: 239-245, 2000.

18. Lira-da-Silva RM, Amorim AM, Carvalho FM, Brazil TK. Escorpionismo na Cidade do Salvador, Bahia, Brasil (1982 2000). In: Resumos do XXXVII Congresso da Sociedade Brasileira de Medicina Tropical, Salvador, 2001.

19. Lopes-da-Silva T. Escorpionismo em Ribeirão Preto, Estado de São Paulo. Notas sobre epidemiologia e profilaxia. Arquivos de Higiene e Saúde Pública. 15: 79-90, 1950.

20. Lourenço WR, Cloudsley-Thompson JL, Cuellar O, Eickstedt VRD, Barraviera B, Knox MB. The evolution of scorpionism in Brazil in recent years. The Journal of Venomous Animals and Toxins 2: 121-134. 1995.

21. Lourenço WR, Cloudsley-Thompson JL. Effects of human activities on the environment and the distribution of dangerous species of scorpion. Envenomings and their treatments. Marcel Mérieux, p. 49-60, 1996.

22. Lourenço WR, Cuellar O. Scorpions, scorpionism, life history strategies and parthenogenesis. Journal of Venomous Animals and Toxins 1: 51-62, 1996.
23. Lwanga SK, Lemeshow S. Sample size determination in health studies. Geneva, World Health Organization, p. 2 e 27, 1991.

24. Maradei-Irastorza I. Scorpion envenomation in Lara State, Venezuela: a historical perspective. Journal of Venomous Animals and Toxins 5:104, 1999.

25. Nicolella A, Marques MG, Torres JB, Barros E. Epidemiologia dos acidentes por animais peçonhentos. In: Nicollela A, Barros E, Torres JB, Marques MG (eds). Acidentes com animais peçonhentos: consulta rápida. Porto Alegre, p.19-25, 1997.

26. Spirandeli-Cruz EF, Yassuda CRW, Jim J, Barraviera B. Programa de controle de surto de escorpião Tityus serrulatus Lutz e Mello 1922, no município de Aparecida, (SP), (Scorpiones, Buthidae). Revista da Sociedade Brasileira de Medicina Tropical 28: 123128, 1995.

27. Statistical Package for the Social Sciences/SPSS. Base 7.0 for Windows. User's Guide. SPSS Inc. 564p, Chicago, IL,1996.

28. Velasco-Castrejón O, Lara-Aguilera R, Alátorre H. Aspectos epidemiológicos y clínicos de la picadura de alacrán en una área hiperendémica. Revista Investigacíón Salud Pública (México) 36: 93-103, 1976. 